\section{Integrative Medicine \\ International}

\title{
Strengthening the Ability of General Hospital Neurology Departments to Treat Neuropsychiatric Diseases and Mental Disorders
}

\author{
Wei Zhu ${ }^{a}$ Weidong Pan ${ }^{b}$ \\ ${ }^{a}$ Department of Neurology, Gongli Hospital, the Second Military Medical University, \\ Shanghai, China; ${ }^{b}$ Department of Neurology, Shuguang Hospital Affiliated to Shanghai \\ University of Traditional Chinese Medicine, Shanghai, China
}

\section{Keywords}

Integrative medicine hospital · General hospital · Integrative therapy · Neuropsychiatric disease $\cdot$ Mental disorders $\cdot$ Comorbidity

\begin{abstract}
Due to the "stigma" of neuropsychological and psychogenic disorders in China, patients with psychological and affective disorders mostly prefer to go to general hospitals or integrative medicine hospitals, while the majority of patients with mental disorders in the department of internal medicine are treated in the department of neurology. At present, there are few physicians with the ability to diagnose and treat neuropsychiatric diseases properly in the neurological department of general hospitals in China, and the diagnosis and treatment experience of mental diseases is insufficient. At the same time, the diagnosis and treatment of comorbid patients with internal diseases and mental disorders are more complicated. The psychology departments in general hospitals or mental health centers also have a limited ability to diagnose and treat comorbid diseases relating to internal medicine and mental disorders together. Therefore, this article reviews the current status of diagnosis and treatment of neuropsychiatric and mental disorders in general hospitals or integrative medicine hospitals.
\end{abstract}

(C) 2018 The Author(s)

Published by S. Karger AG, Basel

With the changes of the global disease spectrum and disease burden, neurological diseases and mental health problems will become the major health problems faced by humans in the 21st century [1]. Many epidemiological studies have found that almost all medical diseases can be accompanied by mental and psychological problems, and the severity of their 
symptoms parallel the extent of the disease and the course of the disease [2, 3]. In the past 40 years, with the development of international psychological medicine, various aspects of psychiatry research in China have also undergone great development. However, in the current social environment, due to the "stigma" of the problem, most patients with neuropsychiatric or mental disorders prefer general hospitals for the first consultation rather than the psychology departments of general hospitals or mental health centers [4]. The neurology or encephalopathy departments (integrative medicine hospital) and various medical departments have become the preferred treatment departments for these patients. At present, due to the lack of training and experience in diagnosing and treating primary mental disorders or mental disorders accompanied by medical diseases, many physicians in China often fail to accurately identify and manage these patients with mental disorders, which can easily result in missed diagnoses, misdiagnoses, delayed treatment opportunity and a waste of medical resources, and can even lead to medical disputes [5].

\section{Characteristics of Patients with Mental Disorders in General Hospitals of China}

Depression, anxiety, and somatization disorders are more common in patients with mental disorder treatment in general hospitals, and most of them are comorbid patients. Depression is one of the most common mental disorders. In early 2017, a World Health Organization (WHO) survey showed that there were 322 million people with depression globally [6], of which the prevalence rate of depression in China reached 4.2\% [7]. Depression has become one of the most important diseases that endanger human health. Foreign data show that about $54-80 \%$ of the patients with depression are treated in the clinical departments of general hospitals and receive certain treatments [8]. However, these data are not very accurate in China. There may be more depressive patients treated in some medical departments, but the proportion of the patients identified and appropriately treated should not reach the international data. The depressive disorders involved here should be divided into 2 types: primary depressive disorders without neurological or other systemic diseases, and other types of depressive disorders with organic or subsequent disorders; the latter can be called secondary depressive disorder or comorbidity. Patients with chronic medical conditions are high-risk groups of depressive disorders $[9,10]$. Some patients or families with a primary depressive disorder can also be psychologically diagnosed with "depression," which can also make diagnosis and treatment easier. The diagnosis of patients suffering from depression in medical medicine is more complicated. There is a causal relationship between depression and medical diseases. Patients and their families are reluctant to believe that they have "psychiatric" diseases, so the diagnosis and treatment ability of doctors is relatively high when patients are diagnosed and treated. For example, the most common neurological disease, Parkinson's disease (PD), is often accompanied by depression. Depression is one of the common non-motor symptoms of PD and can even occur before the onset of PD motor symptoms [11]. The pathological changes of PD are mainly characterized by dopamine depletion in the nigra-corpus striatum system, and dopamine pathways in the midbrain edge and midbrain cortex indirectly affect behavior and cognition. Decreased dopaminergic activity in the central nervous system may cause a decrease in or loss of the feeling of happiness, a decrease in emotion and willpower activity, and affect the concentration of receptors such as serotonin (5-HT) and norepinephrine (NA) in the synaptic cleft of the brain, as well as metabolic cycle pathways leading to depression. These are forms of "endogenous depression," that is, the pathological process of PD is prone to generate depression [12]. Besides, PD patients are sluggish, make certain gestures (postural disorders), suffer from muscle stiffness, tremors, and a mask-like face, slobber, their self-care ability of daily living 
declines, and their "image" is severely impaired, forcing them out of normal social groups, out of society, and shutting themselves off. These depressions, due to somatization symptoms, are forms of "extrinsic depression." Therefore, the factors considered in the treatment of depression in PD patients are more complex than those in simple depression, and it is more difficult to select drugs [13]. Selective serotonin receptor reuptake inhibitor drugs are commonly used as antidepressant drugs. These drugs have more interactions with dopaminergic and anticholinergic drugs used in the treatment of PD and are more complicated to use. Stroke is another type of neuropsychiatric comorbidity with an internal cause (brain damage site and receptor pathway) and an extrinsic cause (disability) jointly leading to depression. The occurrence of depression is related to the stroke site and the disease type. Doctors with experience in neurological and psychiatric diseases at the time of diagnosis and treatment have obvious advantages. Multiple sclerosis, myasthenia gravis, and many other chronic neurological diseases can be accompanied by depression. These diseases may be inadequately diagnosed by a simple neurologist or psychiatrist. Patients with this "comorbidity" choose a neurology department or an encephalopathy department of an integrative hospital instead of going to a mental health center or a psychiatric clinic.

Some studies have shown that organic diseases accompanied by anxiety and somatization disorders are often the most common mental disorders in general hospitals [14]. Because of their complex body complaints, they are not easy to identify and deal with. Patients will go to various departments for repeated visits, resulting in a waste of medical resources, time, and money for them and their families. Due to the characteristics of the anxiety disorder itself, the patient presented with multiple systematic and complex somatization symptoms. More than half of the patients with an anxiety disorder had at least 2 systemic somatic symptoms in the past month, and presented with 5 systematic somatic symptoms in severe cases [15], especially with headache, dizziness, fatigue, chest distress, sleep disorders, and numbness as main complaints. These vivid "symptoms" cannot be localized and qualified with organic lesions. The chief complaint and disease course are fully consistent with the organic diseases, but objective examinations (imaging, electromyography, electrophysiology, etc.) cannot confirm them. An example is the common "angina"-like attack of middle-aged women in the general hospital emergency or cardiology departments. Attacks occur every time when they are angry, involving fatigue, precordial pain accompanied with left-shoulder radiation even involving the upper left limb, profuse sweating, anhelation, and the feeling of impending death. Such patients present to the emergency department many times, but neither repeated electrocardiograms, 24-h dynamic electrocardiogram, cardiac function tests, blood tests for myocardial infarction, a treadmill exercise test, nor repeated coronary angiography will determine the coronary heart disease angina. However, the patient identified typical angina symptoms and was eventually referred to a neurology clinic. The most common complaints in neurology are bulging headache, headache, dizzy giddy vertigo, or dizziness, which are paroxysmal symptoms, but the patients mostly only have "ischemic lacunar lesions in the basal ganglia region" when they are repeatedly examined by head CT, MRI, CT or MRI of the cervical spine, and even CT or MRI angiography, and electroencephalogram. The location of the lesions has nothing to do with the patients' symptoms, but the patients often take the report of "ischemic foci" or "lacunar infarction" as a cause of headache or dizziness, require neurological physicians to treat it, and repeatedly use "blood-activating drugs" and "anticoagulant drugs." Another group of patients will seek multiple gastroscopic reports of "chronic superficial gastritis" from several hospitals to find experts to treat their "stomach pain." Patients consider themselves "early stomach cancer," for fear that experts would not pay enough attention to their complaints and treatment delay, or even undergo gastroscopy once a month. In other patients, there were occasional dizziness-like episodes that lasted for several seconds, but they thought it could be a "brain tumor" or early-stage "brain cancer" 
when searching for symptoms online. There are also some more dramatic examples. After a patient in a unit had been diagnosed with glioma, dozens of people in this unit repeatedly went to the hospital's neurology department for examination because of occasional dizziness, headache, or any other head discomfort. It is imperative for neurological physicians to explain their "head discomfort" and to clearly exclude the possibility of a brain tumor. More seriously affected patients will spend more energy and financial resources to make appointments with professors of neurology in famous hospitals. Patients often choose the medical or neurological department that corresponds to the symptoms. In addition, because of the fear of mental disorders, patients are reluctant to undergo diagnosis such as "psychoneurosis," "somatization disorder," and "anxiety state." They rather go to multiple hospitals or specialists. Patients or their families underline the symptoms, emphasizing that they are caused by an organ, and the psychological disorder is covered by organic diseases of various kinds.

\section{Current Problems in the Treatment of Neuropsychiatric Diseases}

Patients with depression, anxiety, and somatization symptoms, either treated in a general hospital or by a psychiatric specialist, are convinced that their various discomforts are due to organic lesions; there is also a small number of patients who know that they may have depression or an anxiety disorder but still choose to go to a general hospital for treatment because of prejudice against mental disorders, and the complaints at the time of treatment are biased towards specific somatization symptoms, emphasizing symptoms and masking emotions. It takes a lot of time and effort for the doctor to get to the patient's core issues. Therefore, there are several problems in the diagnosis and treatment of these diseases by general hospital non-psychologists and non-psychiatrists. First, doctors of neurology or encephalopathy in general hospitals are not qualified for the diagnosis and treatment of psychiatric diseases; therefore, they cannot quickly and effectively identify organic lesions and somatization disorders due to the lack of diagnosis and treatment skills or inexperience. Second, when a psychiatrist or a general hospital psychologist diagnoses and treats a comorbid patient, the expertise in the diagnosis and treatment of neurological or other medical chronic diseases is lacking, and there is also the risk of misdiagnosis and mistreatment. Third, the use of psychiatric drugs by general hospital non-psychiatrists also has qualification restrictions, and psychiatric specialty drugs are not fully available in general hospitals. In China, there is no strict restriction of the use of selective serotonin receptor reuptake inhibitors and other psychiatric drugs as in foreign countries. However, the lack of a corresponding, standardized training in psychiatric common diseases makes the use of these drugs risky. The diagnosis and treatment qualifications of psychiatric diseases of non-psychiatrists in general hospitals are particularly important because they cannot provide strong defenses in the event of a corresponding medical dispute.

\section{Suggestions for Strengthening the Ability to Diagnose and Treat Neuropsychological and Mental Disorders in Non-Psychiatric Clinical Departments of General Hospitals}

The experts in the field of neuropsychiatry in China and the branch of neurology of the Chinese Medical Doctor Association have fully recognized this issue. The neurology branch of the Chinese Medical Doctor Association has set up the first "neuropsychology and mental disorders special committee" composed of neurological specialists [16]. There is a good correlation between neurology and psychiatry department, but the 2 are not equal. Although 
Zhu and Pan: Strengthening the Ability of General Hospital Neurology Departments to Treat Neuropsychiatric Diseases and Mental Disorders

some general hospitals in China already have departments of neuropsychology or neuropsychiatry, neuropsychology and pediatrics and other comorbidities, most neurologists have limited knowledge of mental illnesses and have not carried out effective clinical and theoretical training. The training of general hospital neuropsychiatrists should be strengthened to establish good general hospital neuropsychiatric diagnosis and treatment abilities, with the following suggestions.

Firstly, training in diagnosis and treatment qualifications for psychiatric diseases in neurology or encephalopathy departments (integrated Chinese and Western medicine hospitals) of general hospitals by physician associations or local health institutions are required, and a neuropsychiatric (neurological) specialty has to be established. In order to prevent patients and their families from refusing to be labeled as "psychiatric" because of "stigma," it is recommended that comorbidity outpatient services in general hospitals avoid names such as "psychosomatic diseases outpatient service," "neurological psychological outpatient service," and "mind diseases outpatient service." These outpatient services are managed by trained and qualified neurologists, because neurological and psychiatric diseases have common anatomical sites - the brain and nerves. Mental illnesses have a similar pathogenesis, such as changes in and interactions between some neurotransmitters. A qualified neurologist has his own professional strengths, and it is relatively easy for him/her to understand and accept the theory of psychiatric diseases. There are many crossroads between the two departments. Neurologists should be trained in general knowledge, qualified certificates should be given for training, and updates of education and assessment should be provided regularly.

Secondly, we should pay attention to the cultivation of the general knowledge of neurologists, including the knowledge of Chinese medicine, and establish a neuropsychiatric (neurological) consultation system in general hospitals. The neurologist who has undergone the standardized training of a physician should have a comprehensive basis of medical disease. The heart, lungs, liver, kidney, and other visceral lesions are prone to cause secondary neurological disorders, resulting in mental disorders. In addition to doing the round in medical departments, physicians should also accumulate clinical experience in the diagnosis and treatment of common surgery, obstetrics and gynecology, and pediatric diseases. Physicians should have more standardized training in neurological diseases and should master good neurological positioning and have qualitative specialty knowledge. Based on this, physicians will have a good knowledge of mental illnesses. In contrast to other countries, China is a country with an ancient civilization emphasizing both the west and the east and has a long history of Chinese medicine and Western medicine. Traditional Chinese medicine has a relatively complete system for the diagnosis and treatment of "consciousness" diseases and believes that "happiness, anger, worry, thought, sadness, fear, and shock" can lead to physical diseases, and physical diseases can also affect emotional changes [17], which is fully compatible with the mind and body comorbidity theory of modern medicine. Therefore, it is recommended that these specialists receive a certain degree of theoretical training in Chinese medicine. When conducting psychological counseling on patients with comorbid conditions, they can use the more moderate vocabulary of traditional Chinese medicine to communicate, and try to avoid the use of "depression," "anxiety disorder," "psychiatric abnormalities," "panic attacks," and other words of modern medicine. At the same time, they should imitate the practices of the neurology branch of the Chinese Medical Doctor Association, establish corresponding professional committees, conduct regular academic communications, continue education, and train professional physicians in the neuropsychiatric field. The emphasis of a comprehensive system of consultation for mental illnesses in general hospitals, especially in the wards using the mental and psychological disease consultation system. The consultation records should be clearly organized, concise, and should include conclusions and operability. 
In particular, the use of psychotropic drugs should be as detailed and standardized as possible [18]. In some large general hospitals, there are more specialized psychiatric departments and even psychiatric specialist wards. These psychiatrists can be trained on a regular basis in neurological diseases and common medical conditions or participate in regular training and continue education at relevant professional institutes. Communication with each other about academic activities can to some extent have an effect on mental and neurological treatment. However, it should be noted that the neurological and psychiatric departments of these hospitals need to coordinate and cooperate to avoid wasting a large amount of medical resources.

Thirdly, the types of antipsychotic drugs available in general hospitals should be reconsidered and strictly controlled by the use of qualified management. The use of drug qualifications is a matter of concern to medical institutions in all countries. Rigorous examination of medical qualifications can avoid medical disputes, and it also involves the use of medical insurance shares. At present, most of the general hospitals in China have partial antidepressant and antianxiety drugs such as sertraline, fluoxetine, venlafaxine, duloxetine, as well as antipsychotic drugs, such as olanzapine, sulpiride and perphenazine. In traditional Chinese medicine or integrated traditional Chinese and Western medicine hospitals, there are some Wuling capsules (乌灵胶囊) and Shugan Jieyu capsules (疏肝解郁胶囊), which contain “antidepression" and "anxiolytic" Chinese herbs or plant medicines. However, the types of medicines are incomplete, and drug use restrictions are not perfect. If the general hospital has more specialized neuropsychiatric training and qualified doctors, it should allow the use of more systematic psychotropic drugs. In addition, the qualifications of Chinese patent medicines can be used for doctors with qualifications in Western medicine to conduct comprehensive diagnosis and treatment of comorbid patients. At the same time, it is also necessary to limit the random use of psychotropic drugs in other departments, avoid wasting medical resources, and generating medical accidents and disputes.

Most patients in nonpsychiatry clinics and patients with spiritual emotion and mental disorders treated in wards of general hospitals are comorbid patients, and some are patients with primary mental disorders who are unwilling to undergo psychiatric specialist treatment, which is related to the social environment of our country. The cultural environment of the surrounding population leads to a bias in the understanding of mental disorders and professional treatment among patients and their families, and there is a "stigma." Therefore, it is also necessary to carry out mental health-related health education and prevention measures for social groups.

"There is no health without mental health" is one of the 21st-century medical treatment emphases put forward by the World Health Organization [19]. The reasonable and effective diagnosis and treatment of psychosomatic diseases should be taken as an important clinical work for a comprehensive hospital in the future. Qualified neurologists in general hospitals should pay attention to psychological and mental disorders when diagnosing and treating physical symptoms of patients, as well as pay attention to comprehensive diagnosis and multidimensional considerations. Together with the hospital they can guarantee the rational use of psychotropic drugs, they can improve the mental health of patients, and thus improve the quality of life of these patients. Comprehensive diagnosis and treatment of patients with mental disorders or comorbidities in general hospitals is a guarantee for the rehabilitation of patients. Therefore, it is necessary to further improve the diagnosis and treatment of depressive disorders and standardized treatment capabilities of nonpsychiatric physicians in future research on mental health services in general hospitals to serve public health in China. 


\section{Acknowledgements}

All experts of the Neuropsychology and Mental Disorders Committee of the Neurological Department Physician Branch of the Chinese Medical Doctor Association assisted in the writing of the article. Professor Peng Xie, the chairman of the Neurological Physician Branch of the Chinese Medical Association, made unremitting efforts for the qualification training of neuropsychiatric specialists and organized and formulated a training program for specialist physicians of neuropsychiatric disorders in general hospitals.

\section{Disclosure Statement}

The authors have no conflicts of interest and declare that they have no further financial disclosures to make.

\section{References}

1 Mohit A: Report of WHO's World Mental Health Survey. Lancet 2006;367:968-969.

$2 \mathrm{Hu}$ J, Xu Y: The clinical survey of internal department in general hospitals. Zhong Guo Xin Li Wei Sheng Za Zhi 1989;3:49-54, 95.

3 Du J, Sun Y, Zhang X, Jiang L: The status of mental disorders in internal departments of general hospitals in China. Zhong Guo Xing Wei Yi Xue Za Zhi 2004;13:115-117.

4 Sattler S, Escande A, Racine E, Göritz AS: Public stigma toward people with drug addiction: a factorial survey. J Stud Alcohol Drugs 2017;78:415-425.

5 Shi-Jie F, Hong-Mei G, Li W, Bin-Hong W, et al: Perceptions of stigma and its correlates among patients with major depressive disorder: a multicenter survey from China. Asia Pac Psychiatry 2017;9. doi: 10.1111/ appy.12260.

6 Jääskeläinen E, Juola T, Korpela H, et al: Epidemiology of psychotic depression - systematic review and metaanalysis. Psychol Med 2018;48:905-918.

7 Liu H, He Y, Wang J, et al: Epidemiology of depression at Traditional Chinese Medicine Hospital in Shanghai, China. Compr Psychiatry 2016;65:1-8.

8 Walker J, Burke K, Wanat M, et al: The prevalence of depression in general hospital inpatients: a systematic review and meta-analysis of interview-based studies. Psychol Med 2018:1-14.

9 Patten SB, Williams JVA, Lavorato DH, et al: Patterns of association of chronic medical conditions and major depression. Epidemiol Psychiatr Sci 2018;27:42-50.

10 Jeuring HW, Huisman M, Comijs HC, et al: The long-term outcome of subthreshold depression in later life. Psychol Med 2016;46:2855-2865.

11 Pan W, Liu J, Chen X, Wang Q, et al: A practical consensus guideline for the integrative treatment of Parkinson's disease in Shanghai, China. Integr Med Int 2015;2:56-62.

12 Sun C, Wang D, Jiang W, Liao W, et al: Quantitative evaluation of Chinese herb medicine in the treatment of sialorrhea and frequent nighttime urination in patients with Parkinson's disease. Evid Based Complement Alternat Med 2017;2017:3045260.

13 Pan W, Yang X, Zhou H: The development of integrative medicine. Integr Med Int 2016;3:96-98.

14 Zhou F, Li F: The clinical investigation of the mental disorders in general hospital. Zhong Guo Lin Chuang Yan Jiu 2011;24:705-706.

15 He Y-L, Zhang L, Liu Z-N, Jia F-J, et al: Prevalence of anxiety disorders among outpatients in general hospitals. Chinese Mental Health Journal 2012;126:165-170.

16 http://www.cnaweb.cn/intro/zhuan-wei-hui-list-0.htm.

17 Liao W, Dang C, Pan W: The brief theory of viscus and its manifestations in traditional Chinese medicine. Integr Med Int 2017;4:13-18.

18 Rayner L, Matcham F, Hutton J, Stringer C, et al: Embedding integrated mental health assessment and management in general hospital settings: feasibility, acceptability and the prevalence of common mental disorder. Gen Hosp Psychiatry 2014;36:318-324.

19 Prince M, Patel V, Saxena S, et al: No health without mental health. Lancet 2007;370:859-877. 